\title{
BMJ
}

\section{Risk of venous thromboembolism in users of oral contraceptives containing drospirenone or levonorgestrel: nested case-control study based on UK General Practice Research Database}

\author{
Lianne Parkin, senior lecturer in epidemiology, ${ }^{1}$ Katrina Sharples, senior lecturer in biostatistics, ${ }^{1}$ Rohini K \\ Hernandez, epidemiologist, ${ }^{2}$ Susan S Jick, director ${ }^{2}$
}

${ }^{1}$ Department of Preventive and Social Medicine, Dunedin School of Medicine, University of Otago,

Dunedin 9054, New Zealand

${ }^{2}$ Boston Collaborative Drug Surveillance Program, Boston University School of Medicine, 11 Muzzey Street, Lexington, MA 02421, USA

Correspondence to: S S Jick sjick@bu.edu

Cite this as: $B M J$ 2011;340:d2139 doi:10.1136/bmj.d2139

\section{ABSTRACT}

Objective To examine the risk of non-fatal idiopathic venous thromboembolism in current users of a combined oral contraceptive containing drospirenone, relative to current users of preparations containing levonorgestrel. Design Nested case-control study.

Setting UK General Practice Research Database.

Participants Women aged 15-44 years without major risk factors for venous thromboembolism who started a new episode of use of an oral contraceptive containing $30 \mu \mathrm{g}$ oestrogen in combination with either drospirenone or levonorgestrel between May 2002 and September 2009. Cases were women with a first diagnosis of venous thromboembolism; up to four controls, matched by age, duration of recorded information, and general practice, were randomly selected for each case.

Main outcome measures Odds ratios and 95\% confidence intervals estimated with conditional logistic regression; age adjusted incidence rate ratio estimated with Poisson regression.

Results 61 cases of idiopathic venous thromboembolism and 215 matched controls were identified. In the casecontrol analysis, current use of the drospirenone contraceptive was associated with a threefold higher risk of non-fatal idiopathic venous thromboembolism compared with levonorgestrel use; the odds ratio adjusted for body mass index was 3.3 (95\% confidence interval 1.4 to 7.6 ). Subanalyses suggested that referral, diagnostic, first time user, duration of use, and switching biases were unlikely explanations for this finding. The crude incidence rate was 23.0 (95\% confidence interval 13.4 to 36.9$)$ per 100000 woman years in current users of drospirenone and 9.1 (6.6 to 12.2) per 100000 woman years in current users of levonorgestrel oral contraceptives. The age adjusted incidence rate ratio was 2.7 (1.5 to 4.7).

Conclusions These findings contribute to emerging evidence that the combined oral contraceptive containing drospirenone carries a higher risk of venous thromboembolism than do formulations containing levonorgestrel.

\section{INTRODUCTION}

In 2002 a series of case reports raised concerns about the risk of venous thromboembolism in users of a recently marketed oral contraceptive containing $30 \mu \mathrm{g}$ oestrogen and a new progestogen, drospirenone. ${ }^{1}$ These reports emerged in the wake of earlier findings that oral contraceptives containing the newer progestogens desogestrel and gestodene ("third generation" pills), and cyproterone acetate, carried higher risks of venous thromboembolism than did pills containing an older progestogen, levonorgestrel. ${ }^{2-7}$

Since 2002 several studies have explored the risk of venous thromboembolism in users of the oral contraceptive containing drospirenone. ${ }^{8-11}$ However, whether this new oral contraceptive carries a higher risk of venous thromboembolism than do preparations containing levonorgestrel remains uncertain. Two industry funded cohort studies, which were done to meet postmarketing surveillance commitments, reported similar risks of venous thromboembolism in users of drospirenone and other oral contraceptives. ${ }^{89}$ However, in one study, the reference group was not clearly defined and may have included women who took oral contraceptives associated with a higher risk of venous thromboembolism. ${ }^{8}$ In the second study, the rates of venous thromboembolism in users of contraceptives containing levonorgestrel were reported to be similar not only to those in drospirenone users but also to those in users of other unspecified oral contraceptives. ${ }^{9}$ This last finding is contrary to what would be expected if that group had included users of higher risk contraceptives. Two other studies found a small increase in risk of venous thromboembolism in users of the drospirenone oral contraceptive when compared with levonorgestrel. ${ }^{1011}$ In a Danish registry based cohort study, the relative risk was 1.64 (95\% confidence interval 1.27 to 2.10$).{ }^{10}$ A population based casecontrol study in the Netherlands reported an odds ratio of 1.7 (95\% confidence interval 0.7 to 3.9). ${ }^{11}$

All four studies included some non-idiopathic cases (women with other known risk factors for venous 
thromboembolism). The presence of risk factors for venous thromboembolism not only affects the probability of the woman developing the condition but also influences decisions about prescribing of oral contraceptives. Therefore, the inclusion of women with such risk factors in the study populations makes drawing valid causal inferences more difficult. ${ }^{12}$ Secondary to this, failure to exclude people with other risk factors for the disease of interest can also result in a dilution of a real effect. ${ }^{13}$ For these reasons, the generally preferred approach in studies of drug safety is to base the investigation on a study population without major risk factors for the disease. ${ }^{12}$ We therefore did a nested casecontrol study to estimate the risk of non-fatal idiopathic venous thromboembolism associated with current use of the oral contraceptive containing drospirenone relative to current use of preparations containing levonorgestrel.

\section{METHODS}

We did a case-control study nested in a cohort of users of oral contraceptives containing $30 \mu \mathrm{g}$ oestrogen in combination with either drospirenone or levonorgestrel. The study was based on data from the UK General Practice Research Database. This database has been used previously in several studies of oral contraceptive use and venous thromboembolism. ${ }^{357}$ It contains information derived from the general practice records of more than three million people in the United Kingdom, including demographic data and details of prescribed drugs, medical diagnoses, hospital admissions, and deaths.

\section{Identification of study cohort}

As drospirenone first became available in the UK in April 2002, we confined the study to the years after this date. We identified all women who had received at least one prescription for an oral contraceptive containing $30 \mu \mathrm{g}$ oestrogen in combination with either drospirenone or levonorgestrel between 1 May 2002 and 30 September 2009 (the study period).

We excluded women if they had a recorded history of risk factors for venous thromboembolism, as well as other conditions that might influence decisions about prescribing of oral contraceptives, such as previous venous thromboembolism, cancer (except nonmelanoma skin cancer), chronic renal failure, myocardial infarction, stroke, other cardiovascular disease, treated hypertension, treated hyperlipidaemia, type 1 diabetes, ulcerative and other colitis, systemic lupus erythematosus, rheumatoid arthritis, ankylosing spondylitis and other spondylopathies, psoriatic arthritis, cystic fibrosis, injecting drug use, and coagulation defects.

To avoid comparing the risk of venous thromboembolism in dissimilar groups of oral contraceptive users, only those women who started a new episode of use of a study oral contraceptive after 1 May 2002 were eligible for inclusion in the study cohort (this included women who had never used oral contraceptives previously, as well as those who had used them in the past and were restarting after a break). We therefore excluded women whose only use of a study oral contraceptive during the study period was part of a continuous episode of oral contraceptive use that had begun before 1 May 2002. We took the date of the first new prescription for a study oral contraceptive after 1 May 2002 as the date of entry into the study cohort.

To estimate woman years of use of drospirenone and levonorgestrel oral contraceptives in the study cohort, we counted the time from the date of the first prescription in each episode of use until the end of the last prescription plus 45 days. We considered consecutive prescriptions for a study oral contraceptive to be part of the same episode of use if the elapsed time between the end date of any one prescription and the issue of the next did not exceed 100 days.

\section{Identification of potential cases and controls}

We carried out all steps in the ascertainment of potential cases and selection of controls without knowledge of the particular study oral contraceptives women were taking.

\section{Cases}

We identified women who had a recorded diagnosis of venous thromboembolism (deep vein thrombosis or pulmonary embolism) after their entry into the cohort from the computer records, using the same Read and OXMIS codes as previously used in studies based on the General Practice Research Database. ${ }^{357}$ We excluded those who did not have a record of treatment with an anticoagulant, as well as any women who continued to receive prescriptions for oral contraceptives after the index date, because the validity of the diagnosis of venous thromboembolism was less certain in such women. We took the date of diagnosis of venous thromboembolism as an index date. Women were eligible for inclusion as potential cases if they were aged 15-44 years on the index date and were current users of a study oral contraceptive. We defined current users as women who received a prescription that would have extended to the index date or to within 30 days of that date. We took current use as the relevant exposure period, because the risk of venous thromboembolism is increased while women are taking oral contraceptives and then returns to normal baseline levels once such use is discontinued. ${ }^{14}$ To be included in the study, current users had to have at least one year of recorded medical information before the index date. We defined the current episode of use as the continuous episode of oral contraceptive use that led up to the index date. This episode could include the use of only one oral contraceptive or the use of more than one if a woman switched directly from one oral contraceptive to another without a break.

Two researchers, blinded to exposure status, independently reviewed the computerised medical records of the potential cases to identify women who had temporary risk factors for venous thromboembolism shortly before the index date. We excluded women who had a record of pregnancy, surgery, major injury, 
or prolonged immobility in the three months before the index date. Any disagreement between the reviewers was discussed and a consensus was reached.

For a subset of the remaining potential cases (the first 42 identified), we sent for copies of hospital discharge letters or outpatient clinic correspondence (as uncomplicated deep vein thrombosis is increasingly being treated in an outpatient setting in the $\mathrm{UK}^{15}$ ) to determine the proportion for whom the diagnosis of venous thromboembolism could be validated and the proportion in whom no other major risk factors for venous thromboembolism were present.

\section{Controls}

For each eligible case, we randomly selected from the study cohort up to four controls matched by year of birth (within two years), number of years of recorded data, and general practice. The index date of each case served as the index date for her matched controls. As with cases, potential controls had to be current users of a study oral contraceptive and to have had at least one year of recorded medical information before the index date. Two researchers, blinded to exposure status, independently reviewed the computer records of potential controls. Controls were subject to the same exclusion criteria as cases.

\section{Statistical analysis}

We used conditional logistic regression to compute odds ratios and 95\% confidence intervals in the casecontrol analyses. The key analysis estimated the risk of non-fatal idiopathic venous thromboembolism in current users of the oral contraceptive containing drospirenone relative to current users of levonorgestrel preparations. To do an analysis stratified according to history of oral contraceptive use (first episode of oral contraceptive use or previous episodes of use), we also did an unmatched analysis but accounted for the matched design by adjusting for year of birth and duration of recorded medical information and adjusting the standard error for clustering on general practice. We used chained equations to impute missing values of body mass index and smoking status ${ }^{16-18}$; we used 100 imputations to obtain stability to one decimal place. The variables included in the imputation model were matching variables (year of birth and duration of recorded information), outcome (deep vein thrombosis, pulmonary embolism, or control), type of oral contraceptive (drospirenone or levonorgestrel), and covariates ( $\log ($ body mass index), age at index date, smoking status, current use of antidepressants, history of varicose veins, history of oral contraceptive use before the current episode, duration of the current episode of use, and whether the current episode involved the use of more than one oral contraceptive).

Potential confounders explored in the analysis models were body mass index (as a continuous variable), history of varicose veins, smoking status (non-smoker, current smoker, past smoker), and antidepressant use. We also explored the effect of the duration of the current episode of oral contraceptive use. We retained adjustment for body mass index in all models; we included other variables if they were significant predictors or if their inclusion altered the odds ratio for the association with type of oral contraceptive by more than $10 \%$. For the key analysis, we present both complete case and imputed data analyses. ${ }^{19}$ We used Stata version 11.0 for all analyses. We estimated incidence rates and age standardised rate ratios by using Stata version 11.0 and Episheet. ${ }^{20}$

\section{RESULTS}

From the computer records, we identified 65 potentially eligible cases of venous thromboembolism. We sent for hospital discharge or outpatient clinic letters for 42 of these women, and we received information for 31. We excluded four women on the basis of this extra information. One had a basilic vein thrombosis that was thought to have an anatomical cause; the remaining three had confirmed venous thromboembolism with predisposing conditions (antiphospholipid syndrome, major trauma, prolonged immobility). For a further two women, the information we received was minimal and we could not determine whether the diagnosis (deep vein thrombosis in one, pulmonary embolism in the other) had been made on the basis of objective tests or if any precipitating events had occurred. We thus included 61 cases in the study; 27 had a diagnosis of deep vein thrombosis alone, and 34 had pulmonary embolism.

Table 1 summarises the characteristics of the 61 cases and 215 controls. The mean age was 32.2 years for the cases and 31.8 years for the controls. Among those with a recorded body mass index (93\% of cases, $86 \%$ of controls), $35(61 \%)$ cases and $57(31 \%)$ controls had a body mass index of 25 or above. The median body mass index was 26.1 (range 19.1-45.7) in cases and 23.3 (17.3-43.2) in controls. Body mass index was unknown for 34 women (four cases); for five of them (all controls), smoking status was also unknown. Those with missing body mass index values were more likely to have had deep vein thrombosis than pulmonary embolism (if cases), be users of levonorgestrel oral contraceptives, be taking antidepressants, be younger, and to be having their first episode of oral contraceptive use. A slightly smaller proportion of cases than controls were current smokers. Three cases and nine controls had a history of varicose veins; one control had a history of superficial venous thrombosis. Cases were more likely to be current users of antidepressants than were controls.

Table 2 shows the results of the key analysis. Seventeen $(28 \%)$ cases and $26(12 \%)$ controls were current users of the drospirenone oral contraceptive. In the matched analysis involving all cases and controls, the unadjusted odd ratio was 3.2 (95\% confidence interval 1.5 to 7.0$)$. The odds ratio adjusted for body mass index was 3.3 (1.4 to 7.6). Further adjustment for a history of varicose veins, smoking, and the use of antidepressants (any antidepressant or tricyclic and non-tricyclic antidepressants separately) had a negligible effect; the odds ratio was 3.1 (1.3 to 7.5). In a model that adjusted for 


\begin{tabular}{|c|c|c|c|}
\hline Characteristic & $\begin{array}{l}\text { Cases } \\
(n=61)\end{array}$ & $\begin{array}{l}\text { Controls } \\
(n=215)\end{array}$ & $\begin{array}{l}\text { Unadjusted matched odds ratio } \\
\qquad(95 \% \mathrm{Cl})\end{array}$ \\
\hline Mean (SD) age (years) & $32.2(7.2)$ & $31.8(7.4)$ & - \\
\hline \multicolumn{4}{|c|}{ Body mass index category $\left(\mathrm{kg} / \mathrm{m}^{2}\right)$ : } \\
\hline$<20$ & $2(3)$ & $27(13)$ & $0.4(0.1$ to 1.7$)$ \\
\hline $20-24.9$ & $20(33)$ & $101(47)$ & 1.0 \\
\hline $25-29.9$ & $20(33)$ & $42(20)$ & $2.4(1.2$ to 4.8$)$ \\
\hline$\geq 30$ & $15(25)$ & $15(7)$ & $5.5(2.1$ to 14.0$)$ \\
\hline Unknown & $4(7)$ & $30(14)$ & $0.7(0.2$ to 2.4$)$ \\
\hline History of varicose veins & $3(5)$ & $9(4)$ & $1.2(0.3$ to 4.4$)$ \\
\hline \multicolumn{4}{|l|}{ Smoking status: } \\
\hline Current smoker & $11(18)$ & $47(22)$ & $0.7(0.3$ to 1.5$)$ \\
\hline Former smoker & $9(15)$ & 39 (18) & 0.7 (0.3 to 1.5$)$ \\
\hline Non-smoker & $41(67)$ & $124(58)$ & 1.0 \\
\hline Unknown & 0 & $5(2)$ & - \\
\hline \multicolumn{4}{|l|}{ Current drug use: } \\
\hline Antipsychotic & 0 & $1(\ll 1)$ & - \\
\hline Any antidepressant & $7(11)$ & $11(5)$ & $2.5(0.9$ to 7.1$)$ \\
\hline Tricyclic antidepressant & $3(5)$ & $2(1)$ & 5.1 (0.8 to 30.4$)$ \\
\hline Non-tricyclic antidepressant & $4(7)$ & $9(4)$ & $1.6(0.5$ to 5.0$)$ \\
\hline Tranexamic acid & $2(3)$ & 0 & - \\
\hline
\end{tabular}

these factors and the duration of the current episode of use, the odds ratio was 3.2 (1.3 to 7.6). These odds ratios from the imputed missing data analysis were slightly higher than those obtained in the complete case analysis (the analysis that excluded cases and controls with missing body mass index and the controls of the excluded cases).

We estimated separate associations for validated and unvalidated cases in a model with an interaction term. After adjustment for body mass index, the odds ratio for validated cases was 4.0 (0.9 to 16.5$)$ and that for unvalidated cases was 3.0 (1.0 to 8.5); the $\mathrm{P}$ value for the interaction was 0.8 . Further adjustment for antidepressant use gave odds ratios of 3.4 (0.8 to 14.9) and 3.4 (1.2 to 10.1).

We also examined the risks of pulmonary embolism and deep vein thrombosis separately; the matched odds ratio for pulmonary embolism (adjusted for body mass index) was 2.1 (0.8 to 5.6). The unadjusted matched estimate for deep vein thrombosis (based on 10 discordant case-control sets) was 8.6 (1.8 to 41.6). Adjustment for body mass index gave an even higher odds ratio with a very wide confidence interval, indicating that the estimate was unreliable in this small subgroup.

We also did matched analyses to examine whether the excess risk of venous thromboembolism in current users of the drospirenone oral contraceptive, relative to users of levonorgestrel, differed according to age ( $<35$ years, $\geq 35$ years); the odds ratios from the interaction model (adjusted for body mass index) were 3.7 (1.3 to 10.7) for the younger women and 2.8 (0.7 to $10.7)$ for the older women; the $P$ value for the interaction was 0.7. Further adjustment for antidepressant use gave odds ratios of 4.1 (1.4 to 12.2 ) and 2.4 (0.6 to 10.2); the $P$ value was 0.6 .

Fourteen (23\%) cases and $36(17 \%)$ controls had no record of any previous oral contraceptive use before the current episode. We did an analysis to explore whether the excess risk of venous thromboembolism associated with the use of the drospirenone oral contraceptive differed according to the history of oral contraceptive use before the current episode (no previous use, at least one previous episode of use). Among first time users, the unmatched odds ratio from the interaction model adjusted for the matching factors and body mass index was 8.6 (1.7 to 42.8 ). For women who had previously used oral contraceptives, the corresponding adjusted odds ratio was 2.4 (1.1 to 5.2 ). The $P$ value for the interaction was 0.17 .

We also did a matched analysis to examine whether the excess risk of venous thromboembolism in current users of the drospirenone contraceptive differed according to the elapsed time since the product had been introduced on to the market. We chose a cut-off date of 1 January 2007 to allow for sufficient woman years of use of both oral contraceptives to be accumulated. We found elevated risks in the periods before and after January 2007 . The odds ratios from the interaction model (adjusted for body mass index) were 2.2 (0.5 to 9.2) in the first period and 4.1 (1.4 to 11.8 ) in the second period; the $\mathrm{P}$ value for the interaction was 0.5 .

Six cases and 17 controls had taken more than one oral contraceptive in the course of their current episode of continuous oral contraceptive use. When we excluded these women, the adjusted (for body mass index) matched odds ratio was 3.2 (1.1 to 8.7). Adjustment for switching (and body mass index), rather than excluding these women, produced similar results; the odds ratio was 3.6 (1.4 to 9.0).

We found 318825 women who met all of the criteria for inclusion in the study cohort. This cohort had an estimated 73853 woman years of use of the oral contraceptive containing $30 \mu \mathrm{g}$ oestrogen and drospirenone and 482229 woman years of use of preparations containing $30 \mu \mathrm{g}$ oestrogen and levonorgestrel. The crude incidence rates were 23.0 (95\% confidence interval 13.4 to 36.9$)$ per 100000 woman years in current users of drospirenone oral contraceptives and 9.1 (6.6 to 12.2 ) per 100000 woman years in current users of levonorgestrel oral contraceptives. The age adjusted incidence rate ratio was 2.7 (1.5 to 4.7 ).

\section{DISCUSSION}

In this study, women who were current users of the oral contraceptive containing drospirenone were about three times as likely to develop non-fatal idiopathic venous thromboembolism as were current users of contraceptives containing levonorgestrel. The matched odds ratio (adjusted for body mass index) in the case-control analysis was $3.3(95 \%$ confidence interval 1.4 to 7.6$)$, and the age adjusted incidence rate ratio in the cohort analysis was $2.7(95 \%$ confidence interval 1.5 to 4.7$)$. 
Table 2 |Current use of oral contraceptives and venous thromboembolism

\begin{tabular}{|c|c|c|c|c|}
\hline $\begin{array}{l}\text { Progestogen in oral } \\
\text { contraceptive }\end{array}$ & $\begin{array}{l}\text { Cases } \\
(n=61)\end{array}$ & $\begin{array}{l}\text { Controls } \\
(n=215)\end{array}$ & $\begin{array}{l}\text { Unadjusted matched } \\
\text { odds ratio }(95 \% \mathrm{Cl})\end{array}$ & $\begin{array}{l}\text { Adjusted matched } \\
\text { odds ratio }(95 \% \mathrm{CI})^{\star}\end{array}$ \\
\hline \multicolumn{5}{|c|}{ Multiple imputation analysist: } \\
\hline Levonorgestrel & 44 & 189 & 1.0 & 1.0 \\
\hline Drospirenone & 17 & 26 & $3.2(1.5$ to 7.0$)$ & $3.3(1.4$ to 7.6$)$ \\
\hline \multicolumn{5}{|c|}{ Complete case analysisł: } \\
\hline Levonorgestrel & 42 & 154 & - & 1.0 \\
\hline Drospirenone & 15 & 22 & - & $2.9(1.1$ to 7.4$)$ \\
\hline
\end{tabular}

*Adjusted for body mass index as continuous variable.

†Missing values for body mass index and smoking are imputed.

fEffect of multiple imputation is illustrated by presentation of complete case analysis adjusted for body mass

index; cases and controls with missing body mass index are excluded from complete case analysis, as are

controls of excluded cases.
Strengths and limitations of study

The study had several strengths. Firstly, we are likely to have identified all potential diagnosed cases of nonfatal venous thromboembolism in the study cohort, because information about outpatient and emergency department visits and hospital admissions in the UK is sent to general practitioners, and the practices contributing to the General Practice Research Database are trained to record the diagnoses associated with such visits and admissions in the patient's record. Secondly, referral and diagnostic biases seem unlikely explanations for our results, as an elevated risk in users of the drospirenone oral contraceptive was also apparent in the analysis confined to cases of pulmonary embolism - cases in whom the severity of the condition makes any potential differential referral of users of the newer oral contraceptive much less likely. Thirdly, for both cases and controls, information about the medical history and prescription of oral contraceptives and other drugs came from medical records and hence was not subject to recall bias. Body mass index also came from records, rather than self report. Controls came from the same general practices as cases and were matched on the number of years of recorded medical information, ensuring comparable quality and duration of information. Moreover, the duration of recorded information was considerable (mean duration 10 years), permitting the ascertainment of comprehensive medical and contraceptive histories. Fourthly, we restricted the study to current users of drospirenone and levonorgestrel contraceptives who received prescriptions from their general practitioners. Although some current users of oral contraceptives could have been excluded from this study because they obtained their contraceptives from other sources such as family planning clinics, the risk of venous thromboembolism in users of the drospirenone contraceptive relative to levonorgestrel users would need to be different in the excluded group for this to have any relevance to the interpretation of our results. Fifthly, confounding of the key results by age, body mass index, smoking, concomitant drug use, or underlying medical conditions is unlikely: we excluded cases and controls with major risk factors for venous thromboembolism, controls were matched to cases for year of birth, and we explored potential confounding in the analysis. We used multiple imputation methods to impute missing data for body mass index and smoking; although this assumes that data are missing at random, nonignorable mechanisms that would have had more than a minimal effect on the estimated odds ratios are unlikely.

We did not have information about family history of venous thromboembolism, but any difference in the prevalence of this risk factor between users of drospirenone and levonorgestrel contraceptives would be unlikely to be of sufficient magnitude to explain a threefold elevated risk. Thus, our results seem very unlikely to be explained by any selective prescribing of the newer product to women at higher risk of venous thromboembolism. Finally, to compare similar groups of users, we confined the study to women who started a new episode of oral contraceptive use after drospirenone was first available for use. We found no evidence that current users of drospirenone as a group included a disproportionate number of short term or first time users (who seem to carry a higher risk of venous thromboembolism ${ }^{21}$ ) than did current users of levonorgestrel pills. Moreover, the excess risk associated with the drospirenone contraceptive persisted after adjustment for duration of use; it was also apparent both in first time users and in women who had taken oral contraceptives previously, and it was lower during the period after the introduction of drospirenone (when it might be expected to be higher if drospirenone users were more likely to be first time users than were women taking levonorgestrel pills) than in later years. The point estimate also remained above 3.0 when we took switching into account.

The main limitation of this study was the relatively small number of cases, which inevitably limited the precision of our estimates of relative risk in the subanalyses, as shown by the wide $95 \%$ confidence intervals. Nevertheless, the point estimates obtained in the analyses stratified by age, certainty of diagnosis, history of contraceptive use, and calendar period were all consistently in the order of at least a twofold to threefold higher risk of venous thromboembolism in users of the oral contraceptive containing drospirenone relative to users of levonorgestrel. Moreover, the results of this study are very similar to those of a larger study that used data from a US claims database. ${ }^{22}$

We did not obtain copies of hospital discharge or outpatient clinic letters for all potential cases, but in the subset of women for whom we had such information all had confirmed venous thromboembolism and only four were considered non-idiopathic. This provides reassurance that virtually all of the women included in this study are likely to be true cases. Although some of the women for whom we did not obtain extra information may have had conditions or events that precipitated their venous thromboembolism, the proportion is likely to be small and, if anything, the inclusion of such women is likely to have resulted in a slight attenuation of the estimates of relative risk. In any event, we found an elevated risk for validated cases. 


\section{WHAT IS ALREADY KNOWN ON THIS TOPIC}

Combined oral contraceptives containing desogestrel, gestodene, and cyproterone acetate are associated with a higher risk of venous thromboembolism than levonorgestrel contraceptives

Limited evidence suggests that an oral contraceptive containing a new progestogen, drospirenone, also carries a higher risk of venous thromboembolism

\section{WHAT THIS STUDY ADDS}

Current use of the drospirenone oral contraceptive was associated with a threefold increase in idiopathic non-fatal venous thromboembolism compared with current use of levonorgestrel pills, although the overall risk was still low

Referral, diagnostic, recall, first time user, duration of use, and switching biases, and confounding, are unlikely explanations for these findings

The crude incidence rates were 23.0 ( $95 \%$ confidence interval 13.4 to 36.9$)$ and 9.1 (6.6 to 12.2) per 100000 woman years in current users of drospirenone and levonorgestrel oral contraceptives

\section{Conclusions and policy implications}

This study adds to emerging evidence that use of the oral contraceptive containing drospirenone is associated with a higher risk of venous thromboembolism than are preparations containing levonorgestrel. Perhaps now is the time for a systematic review on this topic. In the meantime, as no clear evidence exists to show that the use of the drospirenone pill confers benefits above those of other oral contraceptives in preventing pregnancy, ${ }^{25}$ treating acne ${ }^{26}$ alleviating premenstrual syndrome, ${ }^{27}$ or avoiding weight gain, ${ }^{28}$ prescribing lower risk levonorgestrel preparations as the first line choice in women wishing to take an oral contraceptive would seem prudent.

We thank David Skegg and Charlotte Paul for their helpful comments on an earlier draft of the paper and Sheila Williams for help with implementing multiple imputation methods in Stata.

Contributors: LP was involved in the conception, design and conduct of the study, contributed to the analyses, interpreted the results, and wrote the manuscript. SSJ was involved in the conception and design of the study, obtained data for and oversaw the conduct of the study, and contributed to the interpretation of the results and the writing of the manuscript. KS did the imputed data analyses, interpreted the results, and contributed to the manuscript. RKH contributed to the conduct of the analyses, the interpretation of the results, and the manuscript. All authors had full access to all of the data (including statistical reports and tables) in the study and can take responsibility for the integrity of the data and the accuracy of the data analysis. SS) is the guarantor.

Funding: None.

Competing interests: All authors have completed the Unified Competing Interest form at www.icmje.org/coi_disclosure.pdf (available on request from the corresponding author) and declare: no support from any organisation for the submitted work, no financial relationships with any organisations that might have an interest in the submitted work in the previous three years, and no other relationships or activities that could appear to have influenced the submitted work.

Ethical approval: The protocol for this study was approved by the Independent Scientific Advisory Committee for MHRA (Medicines and Healthcare Products Regulatory Agency) database research. Data sharing: If access to the source data used for this study is required please contact the data owner at admin@gprd.com occurred-about $40 \%$ of controls were partners of men with venous thromboembolism, and $60 \%$ were selected through random digit dialling; the index dates for the two groups of controls were the date of diagnosis of venous thromboembolism in the partner and the date of interview, respectively; and the proportion of eligible women who participated was not high.

Our results are also consistent with findings from laboratory based studies in which activated protein $\mathrm{C}$ resistance was shown to be higher in users of the drospirenone oral contraceptive than in users of levonorgestrel preparations and of a similar magnitude to that in users of pills containing desogestrel, gestodene, and cyproterone acetate. ${ }^{2324}$

The investigators in one post-marketing study reported that obese women were differentially prescribed the drospirenone oral contraceptive in their predominantly German study population ${ }^{9}$; however, we found no evidence of such differential prescribing to UK women in our study. In fact, among the controls with a recorded body mass index, a slightly lower proportion of drospirenone users had a body mass index of 25 or above compared with levonorgestrel users $(29 \%$ v 31\%); the median body mass index in the two groups was 22.4 (range 17.9-30.8) and 23.3 (17.3-43.2).
1 Sheldon T. Dutch GPs warned against new contraceptive pill. $B M$ 2002;324:869.

2 World Health Organization Collaborative Study of Cardiovascular Disease and Steroid Hormone Contraception. Effect of different progestagens in low oestrogen oral contraceptives on venous thromboembolic disease. Lancet 1995;346:1582-8.

3 Jick H, Jick SS, Gurewich V, Wald Myers M, Vasilakis C. Risk of idiopathic cardiovascular death and nonfatal venous thromboembolism in women using oral contraceptives with differing progestagen components. Lancet 1995;346:1589-93.

4 Bloemenkamp KWM, Rosendaal FR, Helmerhorst FM, Büller HR, Vandenbroucke JP. Enhancement by factor V Leiden mutation of risk of deep-vein thrombosis associated with oral contraceptives containing a third-generation progestagen. Lancet 1995;346:1593-6.

5 Jick H, Kaye JA, Vasilakis-Scaramozza C, Jick SS. Risk of venous thromboembolism among users of third generation oral contraceptives compared with users of oral contraceptives with levonorgestrel before and after 1995: cohort and case-control analysis. BMJ 2000;321:1190-5.

6 Parkin L, Skegg DCG, Wilson M, Herbison GP, Paul C. Oral contraceptives and fatal pulmonary embolism. Lancet 2000;355:2133-4.

7 Vasilakis-Scaramozza C, Jick H. Risk of venous thromboembolism with cyproterone or levonorgestrel contraceptives. Lancet 2001;358:1427-9.

8 Seeger JD, Loughlin J, Eng PM, Clifford CR, Cutone J, Walker AM. Risk of thromboembolism in women taking ethinylestradiol/ drospirenone and other oral contraceptives. Obstet Gynecol 2007;110:587-93.

9 Dinger JC, Heinemann LAJ, Kuhl-Habich D. The safety of a drospirenone-containing oral contraceptive: final results from the European Active Surveillance Study on oral contraceptives based on 
142,475 women-years of observation. Contraception 2007;75:344-54.

10 Lidegaard O, Lokkegaard E, Svendsen AL, Agger C. Hormonal contraception and risk of venous thromboembolism: national follow up study. BMJ 2009;339:b2890.

11 Van Hylckama Vlieg A, Helmerhorst FM, Vandenbroucke JP, Doggen CJM, Rosendaal FR. The venous thrombotic risk of oral contraceptives, effects of oestrogen dose and progestogen type: results of the MEGA case-control study. BMJ 2009;339:b2921.

12 Jick H, Garcia-Rodriguez LA, Perez-Gutthann S. Principles of epidemiological research on adverse and beneficial drug effects. Lancet 1998;352:1767-70.

13 Rothman KJ, Pool C. A strengthening programme for weak associations. Int J Epidemiol 1988;17:955-9.

14 World Health Organization Collaborative Study of Cardiovascular Disease and Steroid Hormone Contraception. Venous thromboembolic disease and combined oral contraceptives: results of international multicentre case-control study. Lancet 1995;346:1575-82.

15 Winter M, Keeling D, Sharpen F, Cohen H, Vallance P, for the Haemostasis and Thrombosis Task Force of the British Committee for Standards in Haematology. Procedures for the outpatient management of patients with deep venous thrombosis. Clin Lab Haem 2005;27:61-6.

16 Van Buuren S. Multiple imputation of discrete and continuous data by fully conditional specification. Stat Methods Med Res 2007;16:219-42.

17 Raghunathan T, Lepkowski J, Hoewyk J, Solenberger P. A multivariate technique for multiply imputing missing values using a sequence of regression models. Surv Methodol 2001;27:85-95.

18 Royston P. Multiple imputation of missing values: update of ice. Stata J 2005;5:527-36.

19 Sterne JAC, White IR, Carlin JB, Spratt M, Royston P, Kenward MG et al. Multiple imputation for missing data in epidemiological and clinical research: potential and pitfalls. BMJ 2009;338:b2393.
20 Episheet. Spreadsheets for the analysis of epidemiologic data [program]. Episheet, 2005.

21 Kemmeren JM, Algra A, Grobbee DE. Third generation oral contraceptives and risk of venous thrombosis: meta-analysis. $B M J$ 2001;323:131-4.

22 Jick SS, Hernandez RK. Risk of non-fatal venous thromboembolism in women using oral contraceptives containing drospirenone compared with women using oral contraceptives containing levonorgestrel: case-control study using United States claims data. BMJ 2011;340:d2151.

23 Van Vliet HAAM, Winkel TA, Noort I, Rosing J, Rosendaal FR. Prothrombotic changes in users of combined oral contraceptives containing drospirenone and cyproterone acetate. J Thromb Haemost 2004;2:2060-2.

24 Tchaikovski SN, van Vliet HAAM, Thomassen MCLGD, Bertina RM, Rosendaal FR, Morten Sandset P, et al. Effect of oral contraceptives on thrombin generation measured via calibrated automated thrombography. Thromb Haemost 2007;98:1350-6.

25 Maitra NN, Kulier R, Bloemenkamp K, Helmerhorst FM, Gulmezoglu AM. Progestogens in combined oral contraceptives for contraception. Cochrane Database Syst Rev 2009;1:CD004861.

26 Arowojolu AO, Gallo MF, Lopez LM, Grimes DA, Garner SE. Combined oral contraceptive pills for treatment of acne. Cochrane Database Syst Rev 2009;1:CD004425.

27 Lopez LM, Kaptein AA, Helmerhorst FM. Oral contraceptives containing drospirenone for premenstrual syndrome. Cochrane Database Syst Rev 2009;1:CD006586.

28 Gallo MF, Lopez LM, Grimes DA, Schulz KF, Helmerhorst FM. Combination contraceptives: effects on weight. Cochrane Database Syst Rev 2009;1:CD003987.

Accepted: 24 March 2011 